Arthroskopie 2008 $\cdot 21: 221-222$ DOI 10.1007/s00142-008-0467-x

Online publiziert: 15 . Oktober 2008

(c) Springer Medizin Verlag 2008

\author{
R. Becker ${ }^{1}$. C. Fink ${ }^{2}$ \\ ${ }^{1}$ Zentrum für Orthopädie und Unfallchirurgie, Städtisches \\ Klinikum Brandenburg, Brandenburg/Havel \\ 2 Sportsclinic Austria, Innsbruck
}

\title{
Epidemiologie und Konsequenz von Meniskusverletzungen
}

Meniskusverletzungen bilden die häufigste Ursache für operative Eingriffe am Kniegelenk.

Eine Analyse von 10117 Kniearthroskopien mit Meniskuspathologien zeigte, das Männer 2,8-mal häufiger als Frauen betroffen sind $[1,2]$. Akute Verletzungen treten am häufigsten bei Männern im Alter von 31-40 Jahren auf. Meniskusverletzungen bei Frauen sind über alle Altersgruppen gleichmäßig verteilt. Etwa $30 \%$ der nahtfähigen Meniskusverletzungen zeigen als Zweitverletzung eine vordere Kreuzbandruptur.

\section{Akute und chronische Meniskusverletzungen}

Akute Meniskusverletzungen in Kombination mit einer vorderen Kreuzbandruptur betreffen etwas häufiger (56\%) den lateralen Meniskus [3]. Die Meniskusverletzung tritt zum einen bei entlastetem Kniegelenk aufgrund einer kombinierten Außenrotations- und Flexionsbewegung unter Valgusstress, wie z. B. bei der Skiabfahrt, auf [4]. Verletzungen unter Belastung des Kniegelenks, wie beim Fußball, verursachen eher mediale Meniskusrisse.

Chronische vordere Kreuzbandverletzungen zeigen aufgrund der anteroposterioren Instabilität und geringeren medialen Meniskusmobilität in 70\% der Fälle Risse am Innenmeniskus $[3,5,6]$.

Im Zeitraum Januar 1995 bis Dezember 2000 wurden an der Orthopädischen Universitätsklinik Magdeburg 1164 Kniegelenkarthroskopien wegen einer Menis- kuspathologie vorgenommen. Dies entsprach $6 \%$ aller operativen Eingriffe [1]. Eine partielle Meniskusresektion oder -glättung wurden bei 1066 Patienten durchgeführt. Das mittlere Alter dieser Patienten betrug $43 \pm 15$ Jahre. In 98 Fällen (64 mediale und 34 laterale Risse) erfolgte eine Meniskusnaht. Dies entsprach 8,4\% aller operierten Fälle. Das mittlere Alter dieser Patienten betrug 27 \pm 10 Jahre. Die Häufigkeit der Naht von Meniskusrissen ist vergleichbar mit den Erfahrungen von Stone [7], der davon ausgeht, dass gegenwärtig weniger als 10\% der Meniskusrisse genäht werden. Poehling et al. [2] berichten über 6039 Meniskusläsionen, die arthroskopisch beurteilt wurden. Peripherienahe Risse traten dabei in $26 \%$, horizontale Risse in $12 \%$, radiäre Risse in 9,3\%, Lappenrisse in $21 \%$ und komplexe Risse in nahezu 30\% der Fälle auf. Geht man von einer peripheren Rissverletzung von $26 \%$ aus, kann man schlussfolgern, dass wahrscheinlich mehr Meniskusrisse für eine Naht geeignet sind.

\section{Was ist die Konsequenz der Meniskusresektion?}

Eine prospektive longitudinale Studie wies 30 Jahre nach totaler Meniskusresektion im Röntgenbild in 36\% der Fälle signifikante Gelenkspaltverschmälerungen nach [8]. Neyret et al. [9] evaluierten nach mehr als 20 Jahren 167 Patienten, bei denen eine partielle Meniskusresektion vorgenommen worden war. Patienten mit rupturiertem vorderem Kreuzband zeigten in
65\% der Fälle nach 27 Jahren und in 86\% der Fälle nach 30 Jahren radiologische Arthrosezeichen. In einer weiteren klinischen Studie wurden 12 Jahren nach partieller arthroskopischer Meniskusresektion in $48 \%$ der Fälle Zeichen einer Arthrose beschrieben [10]. Über noch höhere Arthroseraten berichteten Scheller et al. [11], die radiologische Gelenkveränderungen bei $65 \%$ der Patienten nach 7 Jahren und bei $87 \%$ der Patienten nach 12 Jahren feststellten. Zusätzlich zeigte sich bei dieser Untersuchung im Lysholm-Score eine Verschlechterung der Patientenaktivität. Das Ausmaß der Meniskusresektion scheint einen direkten Einfluss auf die Arthroseprognose zu besitzen [12].

Tierexperimentelle Untersuchungen bestätigten gleichfalls das erhöhte Arthroserisiko nach einer Meniskusresektion. Little et al. [13] stellten in einem Schafmodell fest, dass bereits 6 Monate nach lateraler Meniskusresektion histomorphologische und immunhistochemische Veränderungen am Knorpel auftraten. Bedenkt man, dass der Außenmeniskus zu ca. 50$70 \%$ und der Innenmeniskus bis zu 50\% für die femorotibiale Lastübertragung verantwortlich sind, scheint der Knorpel diesem mechanischen Anspruch nicht gerecht zu werden $[14,15,16]$. Im Falle einer lateralen Meniskusresektion nimmt die maximale Druckbelastung immerhin um ca. 300\% zu [16].

Basierend auf einer Literaturanalyse von Bellabarba et al. [3] wird angenommen, dass $71 \%$ der medialen und $40 \%$ der lateralen Meniskusläsionen, die mit einer 
akuten vorderen Kreuzbandruptur assoziiert sind, für eine Naht geeignet erscheinen. Bei chronischer vorderer Kreuzbandruptur verringert sich die Nahtfähigkeit auf $54 \%$ am medialen und auf $26 \%$ am lateralen Meniskus. Entsprechend der Klassifikation von Cooper et al. [17] treten $60,7 \%$ der akuten traumatisch bedingten Meniskusläsionen im peripheren Meniskusbereich (Zonen o und 1 ) auf. Zone 2 war bei $37,6 \%$ der Läsionen betroffen. Diese Ergebnisse belegen, dass eine große Anzahl der Meniskusrisse im vaskulären Bereich liegen.

\section{Resektion oder Naht?}

Nimmt man die Daten von Poehling et al. [2] und Cooper et al. [17] zur Grundlage, scheint eine gewisse Diskrepanz zwischen der bisher berichteten Häufigkeit einer Meniskusnaht (10\%) und der Häufigkeit von Läsionen, die als nahtfähig befundet werden (ca. 30\%). Neuere Fadennahtinstrumente wie der Meniscal Clinch ${ }^{\oplus}$ von Arthrex, der FastFix ${ }^{\circledast}$ von Smith \& Nephew oder der MaxFire ${ }^{\curvearrowleft}$ von Biomet werden evtl. dazu beitragen, dass die Meniskusnaht häufiger vorgenommen wird.

Nachdem die essenzielle Bedeutung der Menisken für das Kniegelenk erkannt wurde und klinische Studien die Auswirkung von Meniskusresektionen belegen, scheint das operative Management von Meniskusverletzungen einen Wandel zur Meniskusnaht und damit zum Meniskuserhalt zu erfahren. Die Meniskuschirurgie hat in den vergangenen Jahren deutlich an Bedeutung gewonnen.

Die vorliegende Ausgabe befasst sich mit Grundlagen, Tipps und Tricks sowie klinischen Ergebnissen nach Meniskusnaht. Weiterhin werden alternative Möglichkeiten wie Meniskusersatz und allogene Meniskustransplantate diskutiert.

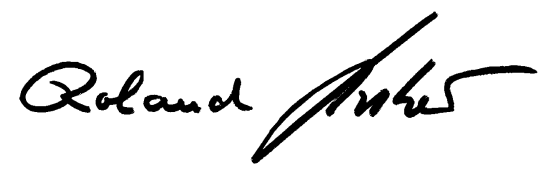

Roland Becker

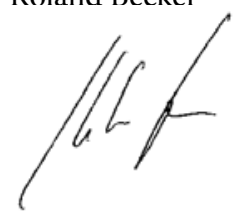

Christian Fink

\section{Korrespondenzadresse}

\section{PD Dr. R. Becker}

Zentrum für Orthopädie und Unfallchirurgie, Städtisches Klinikum Brandenburg Hochstr. 29, 14776 Brandenburg/Havel r.becker@klinikum-brandenburg.de

\section{Literatur}

1. Becker R (2002) Meniskusverletzungen: Untersuchungen zur Meniskusnaht und Meniskusheilung. Habilitationsschrift, S 9-11

2. Poehling GG, Ruch DS, Chabon SJ (1990) The landscape of meniscal injuries. Clin Sports Med 9: 539 549

3. Bellabarba C, Bush-Joseph CA, Bach BR jr (1997) Patterns of meniscal injury in the anterior cruciatedeficient knee: a review of the literature. Am J Orthop 26: 18-23

4. Spondeller P, Beaty GM (1996) Fractures and dislocation about the knee. Ligament injuries. In: Rockwood C, Wilkins KBJ (eds) Fractures in children. Lippincott-Raven, Philadelphia New York, pp 1300-1302

5. Shoemaker SC, Markolf KL (1986) The role of the meniscus in the anterior-posterior stability of the loaded anterior cruciate-deficient knee. Effects of partial versus total excision. J Bone Joint Surg [Am] 68: 71-79

6. Allen CR, Wong EK, Livesay GA et al. (2000) Importance of the medial meniscus in the anterior cruciate ligament-deficient knee. J Orthop Res 18: 109-115

7. Stone KR (1999) Current and future directions for meniscus repair and replacement. Clin Orthop 367: 273-280

8. McNicholas MJ, Rowley DI, McGurty D et al. (2000) Total meniscectomy in adolescence. A thirty-year follow-up. J Bone Joint Surg [Br] 82(2): 217-221

9. Neyret P, Donell ST, DeJour H (1993) Results of partial meniscectomy related to the state of the anterior cruciate ligament. Review at 20 to 35 years. J Bone Joint Surg [Br] 75: 36-40

10. Higuchi H, Kimura M, Shirakura K et al. (2000) Factors affecting long-term results after arthroscopic partial meniscectomy. Clin Orthop 377: 161-168

11. Scheller G, Sobau C, Bulow JU (2001) Arthroscopic partial lateral meniscectomy in an otherwise normal knee: clinical, functional and radiographic results of a long-term follow-up study. Arthroscopy 17: 946-952

12. Englund M, Lohmander LS (2004) Risk factors for symptomatic knee osteoarthritis fifteen to twentytwo years after meniscectomy. Arthritis Rheum 50: 2811-2819

13. Little C, Smith S, Ghosh P, Bellenger C (1997) Histomorphological and immunohistochemical evaluation of joint changes in a model of osteoarthritis induced by lateral meniscectomy in sheep. J Rheumatol 24: 2199-2209

14. Fukubayashi T, Kurosawa $\mathrm{H}$ (1980) The contact area and pressure distribution pattern of the knee. $A$ study of normal and osteoarthrotic knee joints. Acta Orthop Scand 51: 871-879

15. Ahmed AM, Burke DL (1983) In vitro measurement of static pressure distribution in synovial joints, part l: tibial surface of the knee. J Biomech Eng 105: $216-225$
16. Paletta GAJ, Manning T, Snell E (1997) The effect of allograft meniscal replacement on intraarticular contact area and pressures in the human knee. A biomechanical study. Am J Sports Med 25: 692698

17. Cooper DE, Arnoczky SP, Warren RF (1991) Meniscal repair. Clin Sports Med 10:529-548 